\title{
Fractional Excretion of Potassium
}

National Cancer Institute

\section{Source}

National Cancer Institute. Fractional Excretion of Potassium. NCI Thesaurus. Code

C114222.

The determination of the fractional excretion of potassium that is computed based upon the concentrations of potassium and creatinine in both blood and urine. 